\title{
Rationale and Design of the ARREST Trial Investigating Mesenchymal Stem Cells in the Treatment of Small AAA
}

\author{
Short Title: Rationale for the ARREST Trial
}

S. Keisin Wang, MD, Linden A. Green, PhD, Ashley R. Gutwein, MD, Natalie A. Drucker, MD, Raghu L. Motaganahalli, MD, Andres Fajardo, MD, Clifford C. Babbey BS, and Michael P. Murphy, MD

IU Health Center for Aortic Disease

Indiana University School of Medicine, Division of Vascular Surgery, Indianapolis IN

VA Center for Molecular and Cellular Therapeutics in Cardiovascular Disease

Richard L. Roudebush VA Medical Center, Indianapolis IN

\section{Corresponding author:}

Michael P. Murphy M.D.

Associate Professor

Department of Surgery - Division of Vascular Surgery

Indiana University School of Medicine

1801 Senate Blvd MPC\# 2-3500, Indianapolis, IN 46202

Phone: (317) 962-0282

Fax: (317) 962-0289

Email: mipmurph@iupui.edu

\section{Author Contributions:}

SKW, MPM writing and reviewing manuscript, data collection, sample collection

LAG, ARG, RLM, AF, NAD, KEL: sample collection, writing and reviewing manuscript

This is the author's manuscript of the article published in final edited form as:

Wang, S. K., Green, L. A., Gutwein, A. R., Drucker, N. A., Motaganahalli, R. L., Fajardo, A., ... Murphy, M. P. (2017). Rationale and Design of the ARREST Trial Investigating Mesenchymal Stem Cells in the Treatment of Small AAA. Annals of Vascular Surgery. https://doi.org/10.1016/j.avsg.2017.08.044 


\section{Abstract}

\section{Background}

Abdominal aortic aneurysms (AAA) are a major source of morbidity and mortality despite continuing advances in surgical technique and care. Although the inciting factors for AAA development continue to be elusive, accumulating evidence suggests a significant peri-aortic inflammatory response leading to degradation and dilation of the aortic wall. Previous human trials have demonstrated safety and efficacy of mesenchymal stem cells (MSCs) in the treatment of inflammation-related pathologies such as rheumatoid arthritis, graft vs host disease, and transplant rejection. Therefore, herein we describe the ARREST trial, a Phase I investigation into the safety of MSC infusion for patients with small AAA and the cells' effects on modulation of AAA-related inflammation.

\section{Methods}

ARREST is a Phase I, single-center, double-blind, randomized controlled trial (RCT) investigating infusion both dilute and concentrated MSCs compared to placebo in 36 small AAA (35-45 $\mathrm{mm})$ patients. Subjects will be followed by study personnel for 12 months to ascertain incidence of adverse events, immune cell phenotype expression, peripheral cytokine profile, and peri-aortic inflammation. Maximum transverse aortic diameter will be assessed regularly for 5 years by a combination of CT and duplex sonography.

\section{Results}

Four patients have thus far been enrolled, randomized, and treated per protocol. We anticipate the conclusion of the treatment phase within the next 24 months with ongoing long-term follow up.

\section{Conclusion}


ARREST will be pivotal in assessing the safety of MSC infusion and provide preliminary data on

56 the ability of MSCs to favorably modulate the pathogenic AAA host immune response. The data gleaned

57 from this Phase I trial will provide the groundwork for a larger, Phase III RCT which may provide the 58 first pharmaceutical intervention for AAA.

59

60 Clinical Trial Registration: $\underline{\text { https://clinicaltrials.gov/ct2/show/NCT02846883 }}$

61 Unique Identifier: NCT02846883

62

63 Keywords: Inflammation, Mesenchymal Stem Cells, Abdominal Aortic Aneurysm, Vascular Disease 


\section{Introduction}

Each year in the United States, over 200,000 patients are diagnosed with an abdominal aortic aneurysm (AAA). Moreover, 15,000 will die from rupture making it the \#11 killer of Americans per annum. ${ }^{1}$ As a result, over 40,000 highly-morbid aortic operations are performed with health care costs in excess of $\$ 116,000$ over two years after diagnosis. ${ }^{2}$ Although pharmaceuticals such as doxycycline are currently in trial for AAA treatment, no FDA approved medical treatment currently exists for those who do not qualify for operative repair. ${ }^{3}$

While the etiology of AAA formation and growth is unclear at this time, we do know that an intense local inflammatory response leads to the eventual loss of vessel wall integrity. ${ }^{4}$ The therapeutic benefit of mesenchymal stem cells (MSCs) in pathologies with substantial inflammatory responses such as graft vs host disease (GVHD), chronic obstructive pulmonary disease (COPD), and myocardial remodeling after infarction are well established. ${ }^{5-8}$ Previous studies have demonstrated an unique ability of MSCs to secrete $\mathrm{PGE}_{2}$, TGF- $\beta$, and TSG- 6 to stimulate $\mathrm{CD}^{+} \mathrm{CD}^{2} 5^{+} \mathrm{FOXP} 3^{+} \mathrm{T}_{\text {regulatory (Treg) }}$ lymphocytes, which in turn inhibit $\mathrm{CD} 4^{+}$and $\mathrm{CD} 8^{+} \mathrm{T}$-cell proliferation and function. ${ }^{9-11}$ Therefore, the

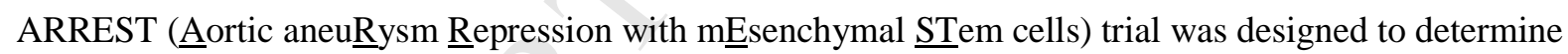
the safety of MSC infusion and provide preliminary data on MSC-induced suppression of AAA-related inflammation. 


\section{Methods}

\section{Synopsis}

ARREST is a single-center, double-blind, RCT that will enroll 36 total patients diagnosed with small AAA (diameter 35-45 mm) by ultrasound or CT. Patients are randomized in a 1:1:1 fashion $(\mathrm{n}=$ 12/group) to receive placebo (Plasmalyte A), $1 \times 10^{6} \mathrm{MSCs} / \mathrm{kg}$, or $3 \times 10^{6} \mathrm{MSCs} / \mathrm{kg}$ through a large bore peripheral IV. After intervention, patients will be followed closely for one year to complete safety and efficacy assessments. Aortic diameter alone will be followed for five years. We expect this trial to identify the most effective dose of MSCs for the modulation of immune cell phenotypes and to elucidate the safety profile of this therapy at various dosages. This information will be utilized to design a larger study and provide power analysis to calculate sample sizes for efficacy.

\section{Recruitment, Randomization, and Blinding}

The trial described in this manuscript adheres to the principles outlined by the Declaration of Helsinki. ${ }^{12}$ ARREST is funded through a VA Merit Award and was approved by the Indiana University Institutional Review Board (IRB). All patients gave informed consent at the time of enrollment. Inclusion and exclusion criteria for enrollment are detailed in Table 1. Non-stratified computer-assisted randomization will be performed for all subjects to fill three cohorts at a 1:1:1 ratio (Figure 1). Unmarked treatment product, prepared by a pharmacist who is not involved in subject assessment or randomization, will be transported in a covered container to the bedside and administered by a study RN and MD concurrently. The PI, RNs, patient, radiologist, and laboratory technicians assessing outcomes will be blinded to treatment group.

\section{MSC Preparation}

Clinical grade allogeneic MSCs will be provided by the Center for Advanced Cellular Therapeutics at the University of Louisville. These MSCs are harvested from bone marrow sourced from 
the iliac crests of healthy young donors. The aspirate is allowed to proliferate in tissue culture. Adherent cells are passaged after reaching $80 \%$ confluency. All therapeutic MSCs will be administered at passage number two to ensure consistency and quality.

\section{Dosage}

Patients randomized to the placebo group will receive an infusion of Plasmalyte A. Subjects in the experimental groups will receive either $1 \times 10^{6}$ or $3 \times 10^{6} \mathrm{MSCs} / \mathrm{kg}$. These dosages were extrapolated from our preclinical models ${ }^{7}$ and are supported by data from previous clinical trials. ${ }^{5,8,13}$

\section{Treatment Administration}

All interventions will be performed at the Richard Roudebush VA Medical Center where continuous telemetry, pulse oximetry, and blood pressure are monitored under the direct supervision of an intensivist, PI, and research RN. Before treatment, all patients will be pre-medicated with hydrocortisone (20-50 mg, IV) and diphenhydramine (25-50 mg, IV). MSC infusions will be delivered through an antecubital vein via a large bore IV (>18 g) at a rate of $2 \mathrm{~mL} / \mathrm{min}$. All patients will be continuously monitored from 15 minutes prior to the start of infusion until two hours after infusion cessation.

\section{Baseline Screening and Follow-Up}

The following evaluations will be carried out at baseline to determine patient eligibility: (1) CBC and CMP; (2) infectious disease panel; (3) medical history and physical exam; (4) medication history; (5) pregnancy test for women of childbearing age; (6) 12-lead ECG; (7) duplex ultrasound of the abdominal aorta. Patients are additionally evaluated on the day of the procedure and in clinic on post-intervention days 3, 7, 14, 30, 180, and 365 (Table 2). At each visit, blood will be obtained for evaluation of cytokine expression (Table 3) and lymphocyte phenotyping by ELISA and FACS analysis respectively. On postintervention days 14 and 365, the patient will undergo an abdominal/pelvis 18-FDG PET/CT. Duplex 
134 sonography of the aorta will be performed annually from years two to five post-infusion to track long-

135 term aortic growth.

136

137

Endpoints

138

Through a regimented office review of adverse events via chart review, history, and physical

139 examination over 1-year follow-up, we plan to test the hypothesis that allogeneic MSCs do not result in

140 significant cardiovascular, respiratory, or infectious complications. Additional endpoints of interest are

141 (1) AAA growth rate by maximum transverse diameter on CT and duplex, (2) number of circulating Treg

142 lymphocytes, (3) cytokine expression in peripheral blood, and (4) changes in the inflammatory AAA

143 environment as assessed by 18 -FDG/PET. 


\section{Discussion}

$$
\text { Currently, the US Preventative Task Force (USPTF) recommends aortic ultrasound screening for }
$$
all males older than 65 who have smoked more than 100 cigarettes in their lifetime or have a family history of AAA. ${ }^{14}$ If diagnosed, observation with serial imaging is recommended until cross-sectional diameter exceeds $5.5 \mathrm{~cm}$. However, if patients with AAA of $3.5 \mathrm{~cm}$ experience a $50 \%$ reduction in the rate of expansion, surgical repair will not be required for 9.5 years which exceeds the lifespan of half of this patient population. ${ }^{15,16}$ As a result, there has been significant interest in the pharmaceutical inhibition of AAA expansion to reduce healthcare costs and improve quality of life. Doxycycline, statins, and propranolol have all shown some promising results in uncontrolled studies, but numerous RCTs have failed to replicate these results. ${ }^{17-20}$

The initial insult which leads to AAA formation and enlargement is unknown but manifests with adventitial recruitment and activation of $\mathrm{CD} 14^{+}$monocytes, which is predominantly observed in the M1 inflammatory phenotype. ${ }^{4}$ The peripheral blood of AAA patients is also rich in $\mathrm{CD}^{+} / \mathrm{CD}^{+} \mathrm{CD} 28^{-}$ cytotoxic T-cells primed to release IFN and perforin. Interestingly, these $\mathrm{CD} 4^{+} / \mathrm{CD} 8^{+} \mathrm{CD} 28^{-}$lymphocytes are more abundant in patients with small $(<4 \mathrm{~cm})$ aneurysms suggesting a more aggressive inflammatory response in early AAA formation. ${ }^{21}$ It is likely that wall tension increasingly becomes the driver for aneurysm growth as sac size enlarges concordant with Laplace's Law. The same cytotoxic T-cells are also overexpressed in $\mathrm{RA}^{22}$ and ankylosing spondylitis ${ }^{23}$ suggesting an autoimmune component to early AAA formation. Deficiency of the transcription factor FOXP3, which affects the immunosuppressive ability of the $\mathrm{CD} 4^{+} \mathrm{CD} 25^{+} \mathrm{FOXP}^{+}$Treg lymphocyte, is a primary cause of autoimmune and inflammatory pathologies in humans. ${ }^{24}$ Not surprisingly, a decrease in Treg differentiation is also observed in the AAA condition compared to risk-factor matched controls. ${ }^{25}$ 
MSCs do not constitutively express MHC-II and therefore largely escape host immune

170 recognition. ${ }^{26}$ Because of this phenomenon, allogeneic MSCs from healthy donors can be administered without rejection to the AAA patient. Previous studies have shown tissue reparative activity after intravenous infusions of MSCs in end organs such as the heart, pancreas, and brain. ${ }^{6}$ Lee et al administered human MSCs in a mouse model of myocardial infarction at the time of injury and found that most of the MSCs became entrapped in the lungs as microemboli. When mRNA from these human cells were isolated from the host, over 50 genes, including potent anti-inflammatory products TSG-6, $\mathrm{PGE}_{2}$, and IL-10, were found to be upregulated. Host alveolar macrophages responded to MSC infusion by increasing TNF- $\alpha$ which, in-turn, caused the MSCs to release their unique secretome and decreased overall infarct size (Figure 2). ${ }^{6}$

In our preliminary studies with the murine AAA topical elastase model, we found that human MSCs can enhance mouse Treg suppressor function (Figure 3), increase aortic tissue Treg counts, and inhibit overall AAA growth. Additionally, when our clinical grade MSCs were cultured with TNF- $\alpha$, a cytokine found in abundance in the systemic circulation of the AAA patient, IL-10, a potent antiinflammatory cytokine, increased 12-fold (Figure 4). Interestingly, an in vivo trial demonstrated that human IL-10 peaked at day 3 when mice were injected with human MSCs at the time of AAA induction but murine IL-10 continued to increase all the way to day 21 , when the experiment was terminated

\section{(Figure 5).}

We hypothesize that MSC administration will increase Treg suppressor function in a dose dependent manner. This effect is likely related to the increase in secreted IL-10, PGE 2 , and TSG-6 from the MSCs sequestered in the host pulmonary system. Treg lymphocytes then increase their antiinflammatory activity by upregulation of IL-10. ${ }^{25}$ Previous studies have demonstrated decreased quantity 
and functionality of Tregs in the AAA condition. ${ }^{25}$ Furthermore, treatment with Treg infusion seemed to abrogate AAA expansion in a murine model..$^{27}$

Acceptable dosages for IV MSC administration were previously described in large randomized human studies evaluating various pathologies. LeBlanc et al reported the mean effective dose in treating steroid resistant GVHD for single dose responders was $1.4 \times 10^{6} \mathrm{MSCs} / \mathrm{kg} .{ }^{4}$ Tan et al found that acute rejection of renal allografts could be prevented with two injections of $1-2 \times 10^{6} \mathrm{MSCs} / \mathrm{kg}$, two weeks apart. ${ }^{8}$ Additionally, Weiss et al treated COPD patients with four monthly injections of $1 \times 10^{8}$ MSCs each, followed his subjects for two years, and noted beneficial therapeutic effect and low complication risk. ${ }^{13}$ In these three studies, a total of 276 patients were treated with IV MSCs - no difference in adverse effects were noted between MSC and placebo treatment in any of the studies.

Understanding the degree of inflammation in small AAAs of the ARREST trial as assessed by hybrid PET/CT will have significant relevance in determining the biological effects of MSCs in attenuating the cytotoxic and proteolytic activity of the immune cell infiltrates. Much of this preliminary work was published by Reeps et al who imaged patients with AAA using 18-FDG/PET and found a significant increase in the standardized uptake value (SUV). Furthermore, high SUV activity correlated with $\mathrm{CD} 68^{+}$macrophage and $\mathrm{CD}^{+}$lymphocyte activity. ${ }^{28}$ The utility of this finding has been helpful to clinicians, who now routinely use PET imaging to diagnose inflammatory aneurysms at high risk of rupture. $^{29}$ 


\section{Conclusion}

214

ARREST is an ongoing Phase I study designed to attain critical safety evidence supporting MSC

216 infusions in patients with small AAAs. Additional endpoints will address a gap in knowledge regarding

217 the bioactivity of MSCs by tracking cytokine response and immune cell populations. The results gleaned

218 from this pilot study will be used to design a larger Phase III RCT which may lead to the first

219 pharmaceutical intervention for AAA. 
Acknowledgement

222 under ClinicalTrials.gov as NCT02846883. 


\section{Disclosure}


1. Ashton HA, Buxton MJ, Day NE, et al. The Multicentre Aneurysm Screening Study (MASS) into the effect of abdominal aortic aneurysm screening on mortality in men: a randomised controlled trial. Lancet. 2002;360(9345):1531-1539.

2. Lederle FA, Johnson GR, Wilson SE, et al. Yield of repeated screening for abdominal aortic aneurysm after a 4-year interval. Aneurysm Detection and Management Veterans Affairs Cooperative Study Investigators. Archives of internal medicine. 2000;160(8):1117-1121.

3. Baxter BT, Matsumura J, Curci J, et al. Non-invasive Treatment of Abdominal Aortic Aneurysm Clinical Trial (N-TA(3)CT): Design of a Phase IIb, placebo-controlled, double-blind, randomized clinical trial of doxycycline for the reduction of growth of small abdominal aortic aneurysm. Contemp Clin Trials. 2016;48:91-98.

4. Blomkalns AL, Gavrila D, Thomas M, et al. CD14 directs adventitial macrophage precursor recruitment: role in early abdominal aortic aneurysm formation. Journal of the American Heart Association. 2013;2(2):e000065.

5. Le Blanc K, Frassoni F, Ball L, et al. Mesenchymal stem cells for treatment of steroid-resistant, severe, acute graft-versus-host disease: a phase II study. Lancet. 2008;371(9624):1579-1586.

6. Lee RH, Pulin AA, Seo MJ, et al. Intravenous hMSCs improve myocardial infarction in mice because cells embolized in lung are activated to secrete the anti-inflammatory protein TSG-6. Cell stem cell. 2009;5(1):54-63.

7. Sharma AK, Lu G, Jester A, et al. Experimental abdominal aortic aneurysm formation is mediated by IL-17 and attenuated by mesenchymal stem cell treatment. Circulation. 2012;126(11 Suppl 1):S38-45.

8. Tan J, Wu W, Xu X, et al. Induction therapy with autologous mesenchymal stem cells in livingrelated kidney transplants: a randomized controlled trial. Jama. 2012;307(11):1169-1177.

9. Roddy GW, Oh JY, Lee RH, et al. Action at a distance: systemically administered adult stem/progenitor cells (MSCs) reduce inflammatory damage to the cornea without engraftment and primarily by secretion of TNF-alpha stimulated gene/protein 6. Stem cells (Dayton, Ohio). 2011;29(10):1572-1579.

10. Spaggiari GM, Capobianco A, Abdelrazik H, Becchetti F, Mingari MC, Moretta L. Mesenchymal stem cells inhibit natural killer-cell proliferation, cytotoxicity, and cytokine production: role of indoleamine 2,3-dioxygenase and prostaglandin E2. Blood. 2008;111(3):1327-1333.

11. Nemeth K, Keane-Myers A, Brown JM, et al. Bone marrow stromal cells use TGF-beta to suppress allergic responses in a mouse model of ragweed-induced asthma. Proceedings of the National Academy of Sciences of the United States of America. 2010;107(12):5652-5657.

12. World Medical Association Declaration of Helsinki: ethical principles for medical research involving human subjects. Jama. 2013;310(20):2191-2194.

13. Weiss DJ, Casaburi R, Flannery R, LeRoux-Williams M, Tashkin DP. A placebo-controlled, randomized trial of mesenchymal stem cells in COPD. Chest. 2013;143(6):1590-1598.

14. LeFevre ML. Screening for abdominal aortic aneurysm: U.S. Preventive Services Task Force recommendation statement. Annals of internal medicine. 2014;161(4):281-290.

15. Hinterseher I, Kuffner H, Berth H, et al. Long-term quality of life of abdominal aortic aneurysm patients under surveillance or after operative treatment. Annals of vascular surgery. 2013;27(5):553-561.

16. Aljabri B, Al Wahaibi K, Abner D, et al. Patient-reported quality of life after abdominal aortic aneurysm surgery: a prospective comparison of endovascular and open repair. Journal of vascular surgery. 2006;44(6):1182-1187. 
17. Baxter BT, Pearce WH, Waltke EA, et al. Prolonged administration of doxycycline in patients with small asymptomatic abdominal aortic aneurysms: report of a prospective (Phase II) multicenter study. Journal of vascular surgery. 2002;36(1):1-12.

18. Investigators PAT. Propranolol for small abdominal aortic aneurysms: results of a randomized trial. Journal of vascular surgery. 2002;35(1):72-79.

19. Meijer CA, Stijnen T, Wasser MN, Hamming JF, van Bockel JH, Lindeman JH. Doxycycline for stabilization of abdominal aortic aneurysms: a randomized trial. Annals of internal medicine. 2013;159(12):815-823.

20. Ferguson CD, Clancy P, Bourke B, et al. Association of statin prescription with small abdominal aortic aneurysm progression. Am Heart J. 2010;159(2):307-313.

21. Duftner C, Seiler R, Klein-Weigel P, et al. High prevalence of circulating CD4+CD28- T-cells in patients with small abdominal aortic aneurysms. Arteriosclerosis, thrombosis, and vascular biology. 2005;25(7):1347-1352.

22. Schmidt D, Goronzy JJ, Weyand CM. CD4+ CD7- CD28- T cells are expanded in rheumatoid arthritis and are characterized by autoreactivity. The Journal of clinical investigation. 1996;97(9):2027-2037.

23. Duftner C, Goldberger C, Falkenbach A, et al. Prevalence, clinical relevance and characterization of circulating cytotoxic CD4+CD28- T cells in ankylosing spondylitis. Arthritis research \& therapy. 2003;5(5):R292-300.

24. Franzke A, Hunger JK, Dittmar KE, Ganser A, Buer J. Regulatory T-cells in the control of immunological diseases. Annals of hematology. 2006;85(11):747-758.

25. Yin M, Zhang J, Wang Y, et al. Deficient CD4+CD25+ T regulatory cell function in patients with abdominal aortic aneurysms. Arteriosclerosis, thrombosis, and vascular biology. 2010;30(9):1825-1831.

26. Ryan JM, Barry FP, Murphy JM, Mahon BP. Mesenchymal stem cells avoid allogeneic rejection. Journal of inflammation (London, England). 2005;2:8.

27. Ait-Oufella H, Wang Y, Herbin O, et al. Natural regulatory T cells limit angiotensin II-induced aneurysm formation and rupture in mice. Arteriosclerosis, thrombosis, and vascular biology. 2013;33(10):2374-2379.

28. Reeps C, Essler M, Pelisek J, Seidl S, Eckstein HH, Krause BJ. Increased 18Ffluorodeoxyglucose uptake in abdominal aortic aneurysms in positron emission/computed tomography is associated with inflammation, aortic wall instability, and acute symptoms. Journal of vascular surgery. 2008;48(2):417-423; discussion 424.

29. Chrapko BE, Chrapko M, Nocun A, Stefaniak B, Zubilewicz T, Drop A. Role of 18F-FDG $\mathrm{PET} / \mathrm{CT}$ in the diagnosis of inflammatory and infectious vascular disease. Nuclear medicine review. Central \& Eastern Europe. 2016;19(1):28-36. 


\section{Table/Figure Legends}

Table 1

Inclusion/exclusion criteria utilized in the recruitment of subjects for ARREST.

Table 2

Follow-up protocol for patients enrolled in ARREST. Baseline labs include CBC with differential, CMP, A1c, PT/INR, HIV, and pregnancy testing where appropriate. Follow-up labs consist of $\mathrm{CBC}$ with differential, CMP, and A1c.

Table 3

Cytokines assayed in the peripheral blood during follow-up.

Figure 1

Schematic of the ARREST trial

Figure 2

MSCs release unique anti-inflammatory secretome in response to inflammatory conditions. Infused MSCs travel through the pulmonary circulation (A) and embolize in the alveolar capillaries. TNF- $\alpha$ released by the host macrophages stimulates the MSCs to release their anti-inflammatory secretome that includes PGE 2 , TSG-6, and TGF- $\beta 1$ (B). PGE 2 , TSG-6, and TGF- $\beta 1$ promote Treg suppressor function that downregulates cytotoxic T-cell activity. Tregs secrete IL-10 that polarizes macrophages from the pro- M1 to the anti-inflammatory M2 phenotype. TSG-6, via ligand binding with CD44, also induces M2 macrophages and inhibits neutrophil migration (C).

Figure 3 


\section{Intravenous administration of human MSCs promote murine Treg suppressor function in}

the mouse AAA model. Using the murine topical elastase AAA model, human MSCs or PBS was injected at the time of elastase application. At 72 hours, the mice were sacrificed. $\mathrm{CD} 4^{+} \mathrm{CD} 25^{-} \mathrm{T}$ responder (Tresp) and $\mathrm{CD} 4^{+} \mathrm{CD} 25^{+} \mathrm{CD} 127^{\text {lo }}$ Treg lymphocytes were harvested. The cells were cultured in varying ratios in plates coated with anti-CD3 and anti-CD28 antibodies. Proliferation was normalized to Tresp alone (control). There was a significant reduction in proliferation with Tregs isolated from MSC treated mice compared to control at $0.5: 1$ and 1:1 ratios (Treg:Tresp). Statistically significant values relative to proliferative arm with no regulatory T cell (0:1) are indicated (one-way ANOVA with Dunnett's multiple comparison test). $* P$-value $\leq .05 . * * * * P$-value $\leq .0001$

\section{Figure 4}

Stimulated MSCs robustly secrete IL-10. Clinical grade MSCs utilized in ARREST were cultured in DMEM supplemented with 5\% human platelet lysate, heparin, and L-glutamate. When MSCs were $90 \%$ confluent, $10 \mathrm{ng} / \mathrm{mL}$ of TNF- $\alpha$ was added. After 24 hours, the supernatant was collected and analyzed by ELISA. MSCs secreted $13.23 \pm 0.25$ (SEM) pg/mL of IL-10 compared to $1.12 \pm 0.31 \mathrm{pg} / \mathrm{mL}$ in untreated cells $(P$-value $<.01)$.

Figure 5

Human MSCs secrete IL-10 early and induce endogenous secretion of IL-10. Using our murine elastase model, we administered $10^{6}$ human MSCs after aneurysm induction and peripheral blood ( $\mathrm{n}=5 /$ time point $)$ was collected. Using ELISA with species specific antibodies, we found that there was an initial surge of human IL-10 peaking at day $3(25.59 \pm 11.1(\mathrm{SEM}) \mathrm{pg} / \mathrm{mL})$ and undetectable by day 7 . Murine IL-10 was produced beginning at day $3(72.23 \pm 3.5 \mathrm{pg} / \mathrm{mL})$ and progressively increased until day $21(436.28 \pm 21.52 \mathrm{pg} / \mathrm{mL})$, when the experiment was terminated. This second surge of IL-10 represents 
an endogenous host response to human MSC administration. $* P$-value $<.05$ compared to control, $* * \mathrm{P}<$ $.01, * * * \mathrm{P}<.001$. 

adhesions of peritoneal organs, elevated ESR, or clinical judgement of investigator.

Mycotic AAA defined as saccular morphology, positive blood culture, fever, or clinical judgement of investigator.

Recent flu vaccine (within six weeks).

History of cancer within the last 5 years, except basal cell skin carcinoma with clean border pathology report. eGFR<30mL/min.

Any condition requiring immunosuppressant medications (e.g. organ transplant, psoriasis, Crohn's, alopecia areata, RA, scleroderma, lupus).

ACS or CHF hospitalization in the last 30 days. HIV or HCV positive, or active HBV.

Significant hepatic dysfunction (ALT or AST greater than 2 times normal).

Any bleeding diathesis defined as an INR $\geq 2.0$ (off anticoagulation therapy), history of platelet count less than 70,000 , or hemophilia.

Pregnant or breast feeding women

Life expectancy less than one year.

Inability to provide written informed consent due to cognitive or language barriers (interpreter permitted).

Presence of any clinical condition that in the opinion of the PI or the sponsor makes the patient not suitable to participate in the study. 


\begin{tabular}{|c|c|c|c|c|c|c|c|c|c|c|}
\hline Evaluation & Baseline & Od & $3 d$ & $7 d$ & $14 d$ & $30 d$ & 180d & $1 y$ & $2 y$ & $5 y$ \\
\hline Informed Consent & $x$ & & & & & & & & & \\
\hline Medical History & $x$ & & & & & & & & & \\
\hline Vitals and Physical Exam & $x$ & $x$ & $x$ & $x$ & & $x$ & $\mathrm{x}$ & $\mathrm{x}$ & & \\
\hline General Labs & $x$ & $x$ & & & & $x$ & $x$ & $x$ & & \\
\hline Infectious Disease Labs & $x$ & & & & & & & & & \\
\hline Immunomodulatory Labs & $x$ & & $x$ & $x$ & & $x$ & $x$ & $\mathrm{x}$ & & \\
\hline EKG & $x$ & $\mathrm{x}$ & & & & $x$ & & & & \\
\hline Randomization & $x$ & & & & & & & & & \\
\hline Abdominal Duplex & $\mathrm{x}$ & & & & & & $x$ & $\mathrm{x}$ & $\mathrm{x}$ & $\mathrm{x}$ \\
\hline Adverse Effect Evaluation & $x$ & $x$ & $x$ & $x$ & & $x$ & $x$ & $\mathrm{x}$ & & \\
\hline
\end{tabular}




\begin{tabular}{|c|c|c|c|c|}
\hline G-CSF & IL-12 (p40) & IL-1 $\beta$ & IL-10 & MIP-1 $\beta$ \\
\hline GM-CSF & IL-12 (p70) & IL-2 & IL-17 & TNF- $\beta$ \\
\hline IFN- $\alpha 2$ & IL-17 & IL-4 & IL-23 & VEGF \\
\hline IFN- $\gamma$ & IL-1ra & IL-6 & RANTES & TGF $\alpha$ \\
\hline MCP-1 & IL-1 $\alpha$ & IL-8 & MIP-1 $\alpha$ & TNF- $\alpha$ \\
\hline PDGF-AA & PDGF-AB/BB & Type III Procollagen & $\mathrm{PGE}_{2}$ & TSG-6 \\
\hline MMP-2 & MMP-9 & TGF- $\beta 1$ & Perforin & \\
\hline
\end{tabular}




\section{Patients assessed for eligibility}

Plasmalyte $A$

$$
(n=12)
$$

Randomized to

Treatment

$$
(n=36)
$$

$1.0 \times 10^{6} \mathrm{MSCs} / \mathrm{kg}$.

$$
(n=12)
$$

$3.0 \times 10^{6} \mathrm{MSCs} / \mathrm{kg}$.

$$
\text { ( } n=12 \text { ) }
$$



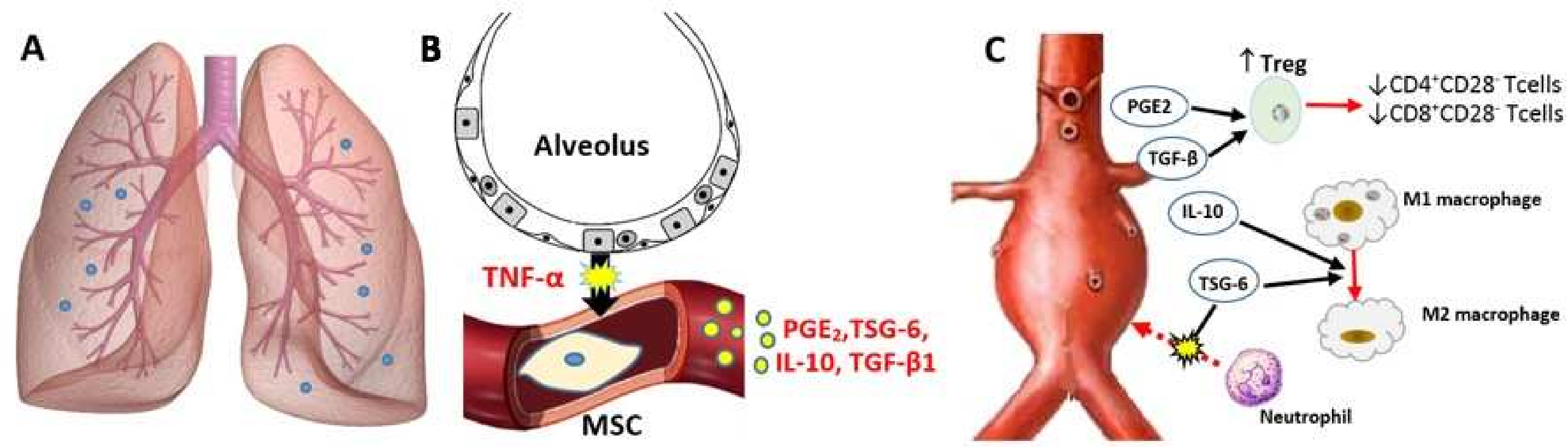


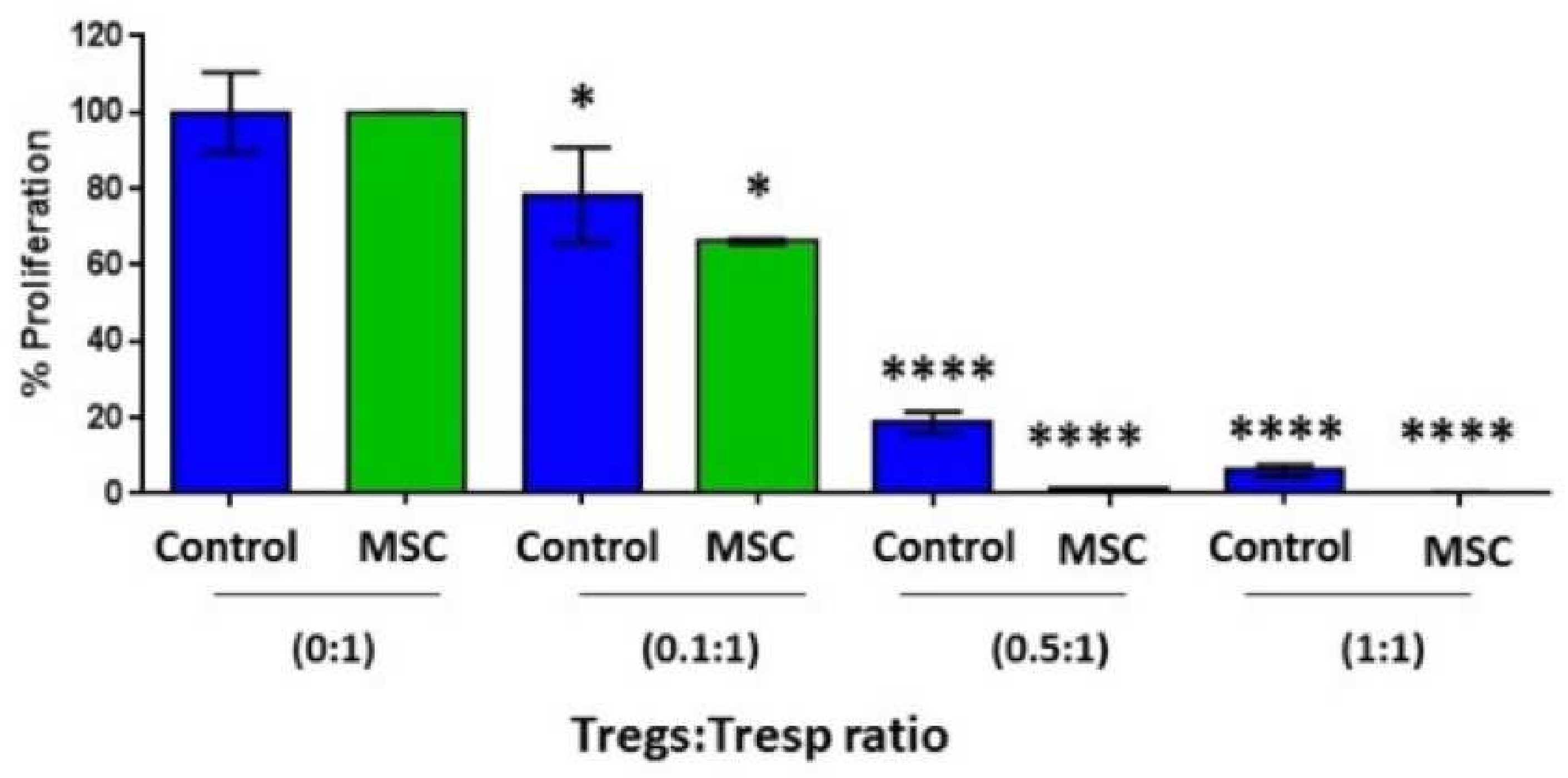




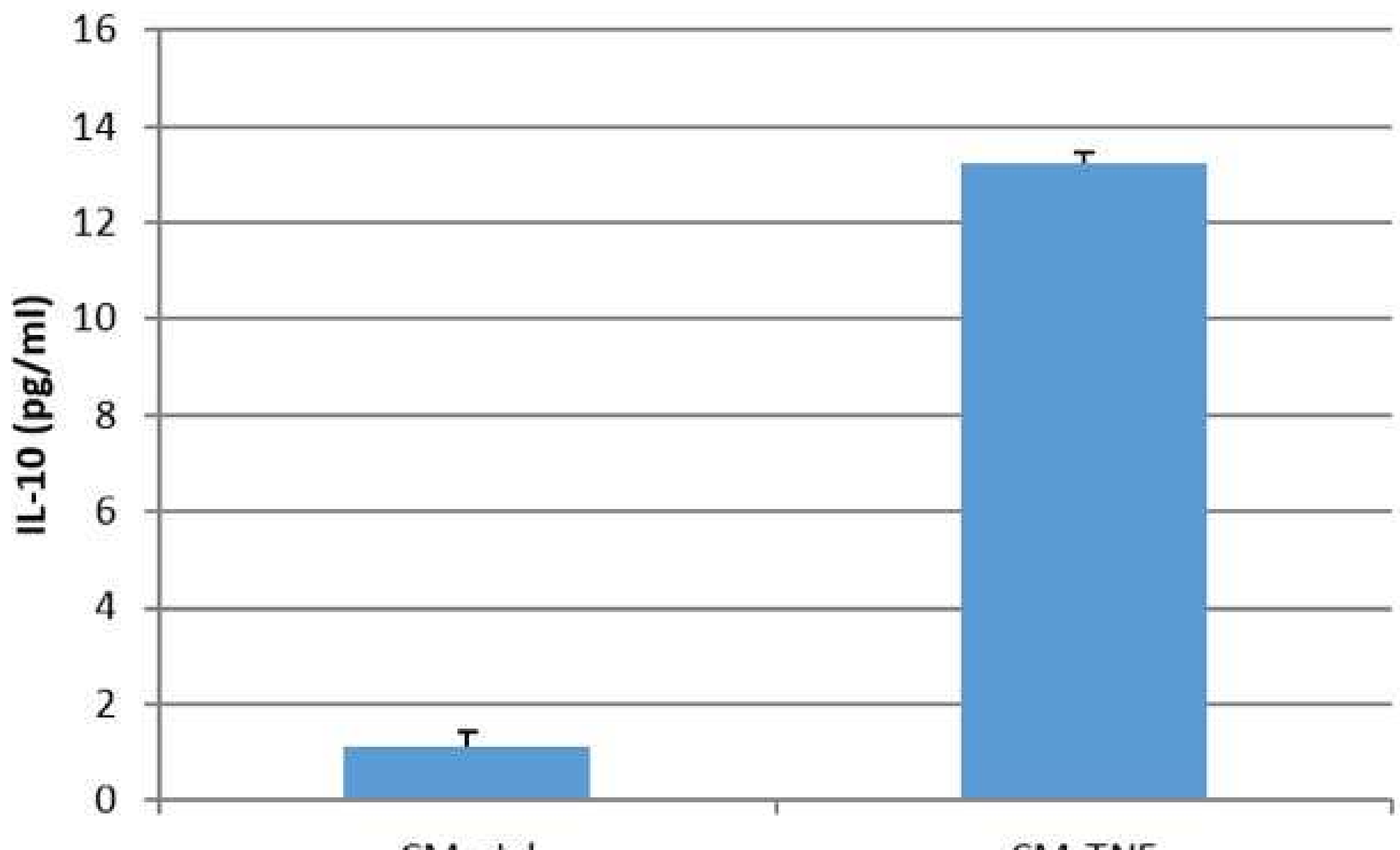

CM-ctrl

CM-TNF 


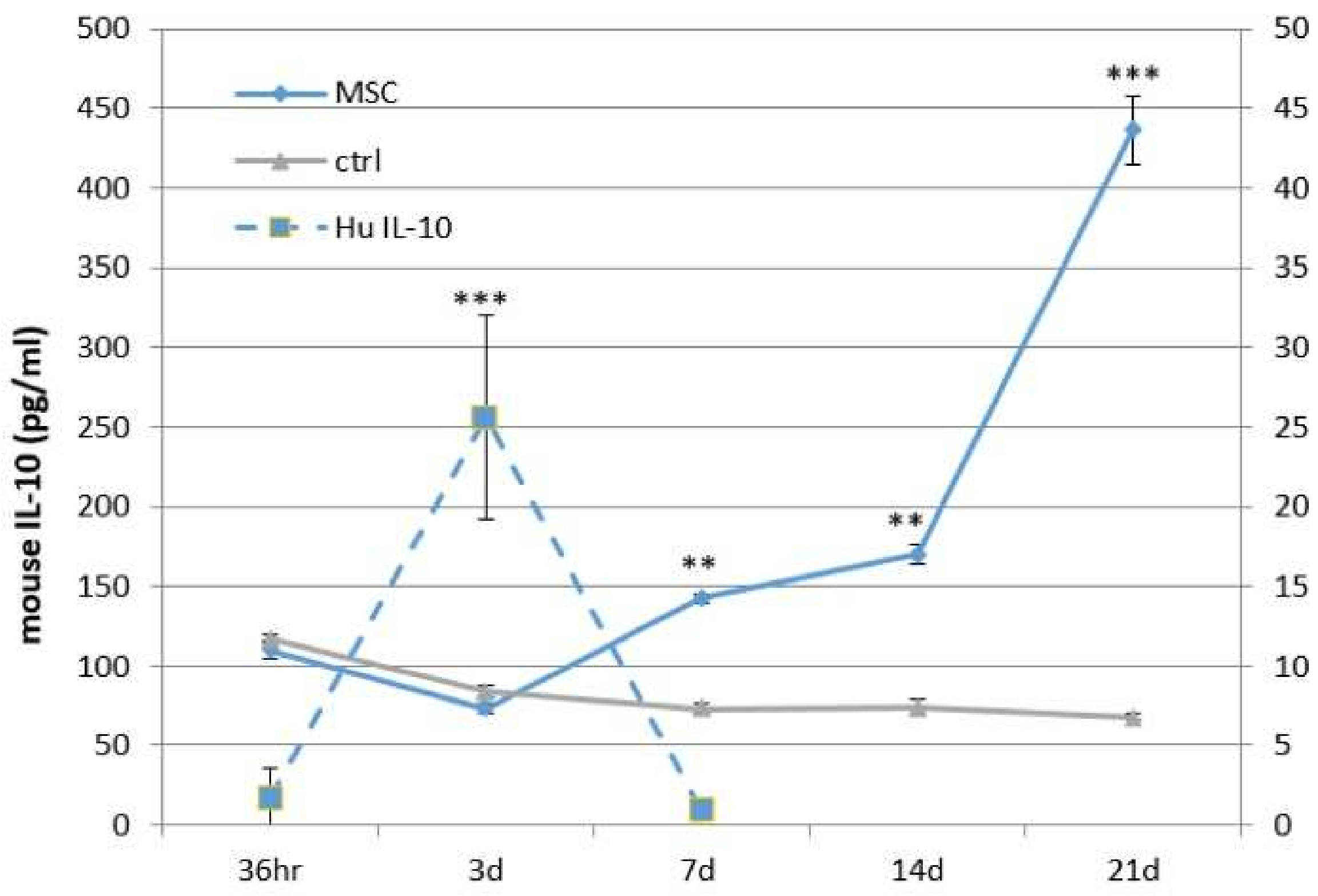

है 